\title{
A Force-Driven Granular Model for EMG based Grasp Recognition
}

\author{
Yinfeng Fang, Dalin Zhou, Kairu Li, Zhaojie Ju and Honghai Liu*
}

\begin{abstract}
It is a challenge to precisely predict hand grasps based on EMG signals given practical scenarios, due to its inherent nature. This paper proposes a solution to tackle the challenge with a force-driven granular model (FDGM).The problem of $n$ class hand grasp classification has been represented as forcebased granular modelling, in which a number of granules are constructed for each class relying on the synchronically captured grasping force. A rule based mechanism is formed for granule generation of each class, and a cross-testing algorithm is proposed to optimise the number of granules. The experiment based on 8case grasp recognition reveals that the proposed method performs better in terms of motion recognition accuracy of multiple EMG channel combination, and is more insensitive to signal interferences. In comparison with other rules of information granulation, it is confirmed that the force-driven rule is of the most efficiency with comparable classification accuracy. The research outcomes pave the way for real-time prediction of grasps and corresponding force in human-centred environments.
\end{abstract}

\section{INTRODUCTION}

Surface EMG based grasp recognition can be utilised to facilitate smart prosthetic hand control. Pattern recognition has achieved high accuracy in the classification of hand movements, however suffering from some practical issues, and one of which is to distinguish hand grasp with dynamic grasping force. Dynamic muscle contraction is usually considered as a negative factor for EMG based hand motion recognition. Till now, the only solution is to train classifiers by data sets comprising varying muscle contraction levels.

Pattern recognition classifies repeatable patterns of EMG activities into discernible classes. The EMG pattern of a grasp at different force levels is somewhat inconsistent, and therefore it presents challenges to pre-trained classifier [1]-[5]. Dynamic muscle contraction significantly influences pattern recognition based prosthetic hand control. As suggested by Scheme et al. [1], the presence of contractions from unseen force levels increased the error considerably by greater than $32 \%$. To counteract the severe degradation, they recommended to form training sets comprising all force levels, which is consistent with the solution proposed by Al-Timemy et al., [2]. Besides, traditional EMG feature varies with muscle contraction level, and $\mathrm{He}$ et al., [3] resorted to identify new features that immunise to muscle contraction.

The paper proposes a supplementary solution to tackle grasp recognition by means of a granular model, where

Yinfeng Fang, Dalin Zhou, Kairu Li and Zhaojie Ju are with the Intelligent Systems and Biomedical Robotics Group, School of Computing, University of Portsmouth, Portsmouth, PO1 3HE, U.K. y infeng fang@gmail. com

Honghai Liu, corresponding author, is with the Intelligent Systems and Biomedical Robotics Group,School of Computing, University of Portsmouth, Portsmouth, PO1 3HE, U.K. honghai. liueport.ac.uk. dynamic muscle contraction is considered. The proposed granular model is originated from the the concept of granular computing ( $\mathrm{GrC})$. GrC solves problems via making use of granules, i.e. groups, classes or clusters of a universe [6]. GrC is very essential to the problem solving strategy of human being, and could generate significant impact on the design of intelligent systems. At present, granular computing is more a theoretical perspective than a coherent set of methods or principles. This paper verifies the impact of $\mathrm{GrC}$ in the field of pattern recognition with a specific case study: EMG based grasp recognition.

Recent years, GrC has started to penetrate into the field of machine learning for classifier development. Liu et al. [7] proposed a granular computing classification algorithm based on distance measurement, where granules are constructed according to geometric shapes. Roh et al. [8] proposed a methodology for designing granular fuzzy classifiers based on information granularity. These classifiers split the entire input space into a collection of subspaces. Both studies aim to design whole classifiers based on the concept of information granulation to achieve equivalent or better performance. However, without sufficient comparison and theoretical proof, the performance is still in doubt. More meaningfully, information granulation be employed to extend any conventional classifiers, like linear discriminant analysis (LDA) and support vector machine (SVM). In 2004, Yuchun et al. [9] built a granular SVM classifier by building a sequence of information granules in advance. Not explicitly using the concept of $\mathrm{GrC}$ or information granulation, Zhu et al. [10] published a paper on Subclass Discrimination Analysis, where subclass (granule) generation and LDA classification are theoretically merged.

As far as information granulation is concerned for pattern recognition, two aspects should be balanced: classification accuracy and model training complexity, which arise two basic questions. Firstly, how to construct granules from a universal data set? Secondly, how to determine the number of granules? A general criterion for granule construction is to draw elements with indistinguishability, similarity, proximity or functionality together, while to diffuse elements to different granules according to their distinguishability, dissimilarity and inconsistence [11]. Specifically, researchers tend to use clustering based algorithms, such as (Nearest Neighbor) NN [10], k-means [12], [13], hierarchical clustering [14] and spatial partition trees [15] to construct granules. The number of granules is closely related to the complexity of model training and classification accuracy. Intuitively, model training complexity would increase with the number of granules. Therefore, 


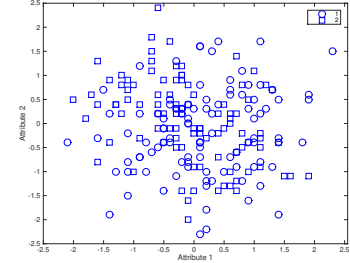

(a) Original data set

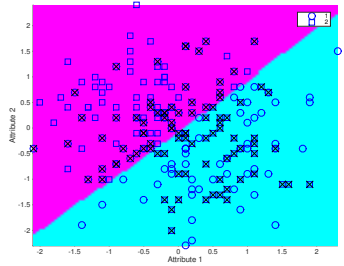

(b) LDA classification result

Fig. 1: The classification result by a LDA classifier

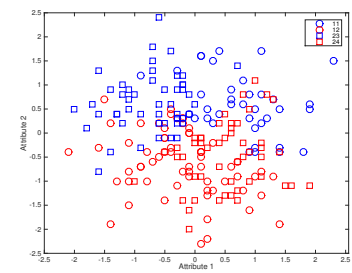

(a) The result of k-means granula- (b) Classification with granular tion

Fig. 2: The classification result by a granular model based LDA classifier using k-means for granulation

solutions should be provided to determine the number of granules in a specific application.

The remaining part of this paper is organised as follows. Section II generalises the granular model for pattern recognition and provides an experiment with a synthetic data set. Section III describes the FDGM. Section IV proposes an algorithm to optimise the number of granules. Section $\mathrm{V}$ demonstrates the protocol for data set collection, feature extraction and the implementation of FDGM. Section VI discloses the experimental results. Section VII concludes the paper.

\section{A Granular Model for Pattern Recognition}

The granular model is defined in this section, and confined to the following notation. Given a data set $\mathcal{D}$ of $K$ training samples (patterns) from $l$ separable classes: $\mathcal{D}=$ $\left\{\left(\mathbf{x}_{k}, y_{k}\right), k=1, \ldots, K\right\}$, where $\mathbf{x}_{k} \in \boldsymbol{R}^{n}$ and $y_{k}$ labels $\mathbf{x}_{k}$ to one of classes in $y_{k} \in \Omega_{c}=\left\{c_{1}, c_{2}, \ldots, c_{l}\right\}$. A traditional classifier is a function: $f: \mathbf{x} \rightarrow \Omega_{\mathbf{c}}$ that assigns a class label $c \in \Omega_{c}$ to a pattern described by a set of attributes $\mathbf{x}=\left\{x_{1}, x_{2}, . . x_{n}\right\} \in \mathbf{R}^{n}$.

According to the theory of information granulation, one class can be decomposed into $m$ subclass granules. Rules for granular decomposition can be customised according to different applications. In despite of any granulation rule, the granular model maps the original data set to a new one with more classes, also called subclass granules. The new data set can be denoted by $\mathcal{D}^{\prime}=\left\{\left(\mathbf{x}_{k}, y_{k}^{\prime}\right), k=1, \ldots, K\right\}$, where $y_{k}^{\prime}$ labels $\mathbf{x}_{k}$ to one of $l \times m$ classes in $\Omega_{c}^{\prime}=\left\{\Omega_{c 1}, \Omega_{c 2}, \ldots, \Omega_{c l}\right\}$ and $\Omega_{c i}=\left\{c_{i 1}, c_{i 2}, \ldots, c_{i m}\right\}(i=1,2, \ldots, l)$ is a set of granules decomposed from the entirety $c_{i}$. The relationship between $c_{i j}$ and $c_{i}$ can be denoted by $c_{i j} \leq c_{i}$, saying $c_{i j}$ is a granule of $c_{i}$. Accordingly, a classifier $f^{\prime}: \mathbf{x} \rightarrow \Omega_{\mathbf{c}}^{\prime}$ can be trained to assign a class label $c^{\prime} \in \Omega_{c}^{\prime}$ to any input pattern $\mathrm{x}$. Therefore, the original $m$-class classification problem upgrades to a $l m$ class problem. After obtaining the prediction result from $f^{\prime}$, an inverse procedure should be applied to $c^{\prime}$ using the relationship $c_{i j} \leq c_{i} . c_{i j}$ is a refinement of $c_{i}$ and $c_{i}$ is a coarsening of $c_{i j}$ [16]. It can be described by a coarsening function, $c_{i}=\operatorname{coarsen}\left(c_{i j}\right)$.

As described above, the granular model solves a $l$-class classification problem by upgrading it to a $l m$-class classification problem, and $m$ granules are generated for each class. This paper utilises a synthetic data set to demonstrate the advantage of the granular model. The data set was randomly generated in accordance of 2-dimensional normal distribution with the same covariance matrix $\Sigma=\left[\begin{array}{cc}0.5 & 0 \\ 0 & 0.5\end{array}\right]$ but different mean vectors. Half of samples labeled as $c_{1}$ are randomly generated with the mean vector $\mu=\left[\begin{array}{l}0.5 \\ 0.5\end{array}\right]$ and the other half are under $\mu=\left[\begin{array}{l}-0.5 \\ -0.5\end{array}\right]$. These samples in class $c_{1}$ are noted by circles in Fig. 1(a). Samples labeled as $c_{2}$ are generated by $\mu=\left[\begin{array}{c}0.5 \\ -0.5\end{array}\right]$ and $\mu=\left[\begin{array}{c}-0.5 \\ 0.5\end{array}\right]$ in half, represented by squares in Fig. 1(a).

Fig. 1(b) demonstrates the self-classification result $(54 \%$ accuary) using LDA classifier, and the incorrectly classified sample are denoted by the symbol ' $x$ '. This result indicates that LDA is not able to solve the classification problem with the given data set, because it is not linearly separable. Fig. 2(a) shows the granulation result for each class by k-means clustering algorithm, where the number of granules is set to 2 . Accordingly, class $c_{1}$ is divided into two granular classes $c_{11}$ and $c_{12}$, while $c_{2}$ is separated into $c_{21}$ and $c_{22}$. In Fig. 2(a), blue circles, red circles, blue squares and red squares represent the samples in $c_{11}, c_{12}, c_{21}$ and $c_{22}$, respectively. Obviously, the original 2-class classification problem becomes 4-class problem after applying the granular model. Consequently, the LDA is trained to predict one of four granular classes $c_{11}$, $c_{12}, c_{21}$ and $c_{22}$. Finally, the coarsening function is applied to recover the prediction results to 2 classes $c_{1}$ and $c_{2}$. As demonstrated in Fig. 2(b), the dividing border divides the space into four parts, and achieves better self-classification accuracy of $64.88 \%$.

\section{Force Driven Granular Model}

Basic issues in information granulation involves two aspects: the construction and the computation of granules. For the construction of granules, granular models should be firstly determined to check if two elements should be gathered to the same granule. Traditional clustering algorithms, like NN, k-means algorithm and Gaussian mixture model (GMM), can be used as the granular model. They assemble homogenised samples into a granule based on their similarity and geometry distance. However, these models are lack of semantic interpretation towards the generated granules. Therefore, FDGM is proposed in this paper to compensate its disadvantage in the case of grasp recognition. The FDGM employs rule based mechanism for the construction of granules as described below. 
The recorded data set contains two parts: EMG signal and force signal. The observations in class $i$ is indicated by a set $\left\{\mathbf{F}_{i 1}, \mathbf{F}_{i 2}, \ldots, \mathbf{F}_{i n_{i}}\right\}$, where

$$
\mathbf{F}_{i j}=\left\{f_{i j}, \mathbf{x}_{i j}\right\}
$$

where $f_{i j}$ is the force variable and $\mathbf{x}_{i j}=\left\{x_{i 1}, x_{i 2}, \ldots x_{i n}\right\}$ is the EMG feature vector. $\mathbf{x}_{i j}$ can be assigned into $K$ different granules by a crisp rule as follows,

$$
\begin{gathered}
f_{i j}-f_{(i, \text { min })} \leqslant s \quad \Rightarrow \quad \mathbf{x}_{i j} \in g_{i 1}, \\
(k-1) s<f_{i j}-f_{(i, \text { min })} \leqslant k s \quad \Rightarrow \quad \mathbf{x}_{i j} \in g_{i k}
\end{gathered}
$$

and

$$
f_{i j}-f_{(i, m i n)}>(K-1) s \quad \Rightarrow \quad \mathbf{x}_{i j} \in g_{i K},
$$

where $g_{i k}$ is the $k^{\text {th }}$ granule, $f_{(i, \text { min })}$ is the minimum force for the observations in class $i$, and $s=\frac{f_{(i, \max )}-f_{(i, \min )}}{K}$, where $f_{(i, \max )}$ is the maximum force for class $i$. As a result, EMG feature vectors in a granule represents the same range of grasping force. In the granule of $g_{i k}$, the bigger value $k$ is, the larger grasping force being reflected by the EMG feature. It is the semantics of the granule, which paves the way of force estimation in our future studies.

\section{DETERMinAtion OF THE NUMBER OF GRANULES}

The most convenient criterion for the selection of the optimal number of granules is the leave-one-out-test, using all but one sample for training and test the sample left out. It is a direct solution, but computationally expensive, especially when the number of samples $(n)$ and classes $(m l)$ are large. Therefore, the 10-fold-cross-test is utilised in this study instead.

For each possible value of $M$, we implement 10-fold-crosstest to obtain the classification accuracy $R_{M}$ to obtain the optimal value $M_{o}$ by solving the following problem

$$
M_{o}=\underset{M}{\operatorname{argmax}} R_{M},
$$

and the proposed algorithm is described in Algorithm 1.

\section{Data Capturing and Signal Processing}

Traditional EMG based grasp recognition involves several important issues: 1) EMG data set recording, 2) EMG feature extraction, 3) classifier training, and 4) system evaluation. Data set recording is to record EMG signals that reflect diverse hand grasps. EMG features are extracted from EMG signals to reduce its randomness and highlight the distinguishability and similarity among EMG patterns. Classifier training functions to optimise the coefficients according to the training data set. System evaluation is to investigate the performance of a tuned system, mainly on the accuracy and stability. This paper aims to improve the performance of a grasp recognition system, in terms of the accuracy and stability. Sensitivity analysis is utilised to evaluate system stability by considering some very practical issues, such as power line noise and white gaussian noise interferences.

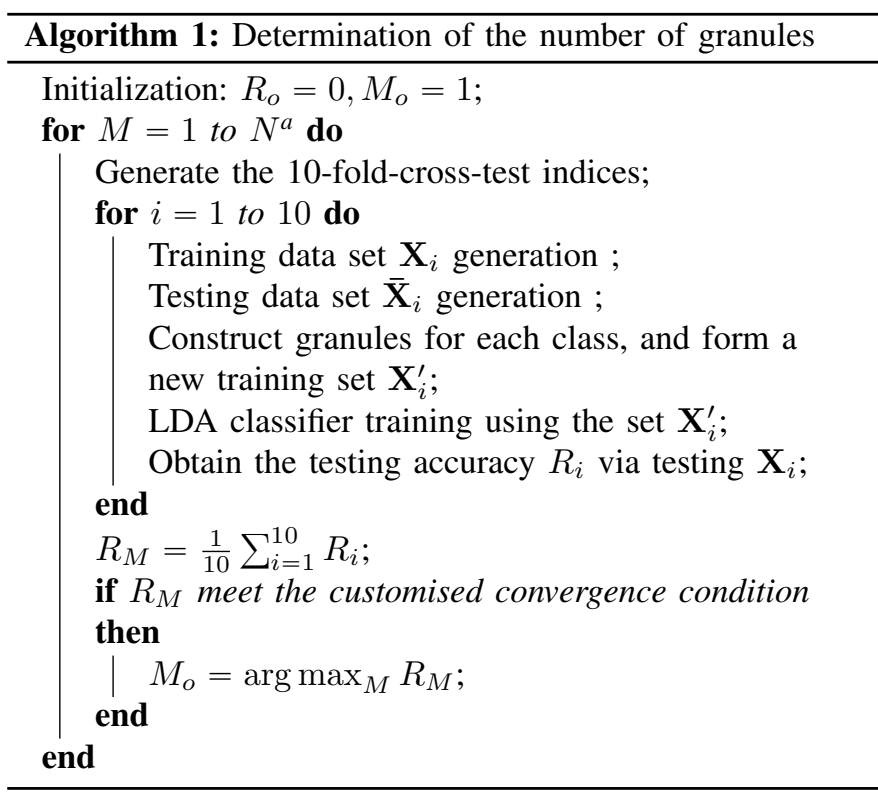

${ }^{a}$ The maximum number of granules. It is determined by the time complexity of the prediction algorithm. The value is set to 80 in experiment to meet the real-time requirement for EMG based grasp prediction.

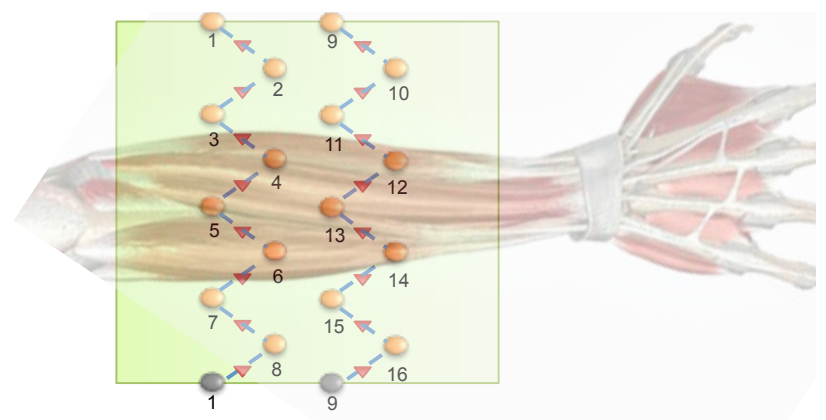

Fig. 3: 16-channel electrode configuration on the forearm.

It has been pointed out that muscle contraction could become a negative factor for a EMG based pattern recognition systems [1]-[5], if it is not properly sorted. We captured EMG signal together with the grasp force during the phase of data set acquisition. Therefore, force signal can be utilised as an additional information in the classifier training stage, though only EMG signal is used for testing late on.

\section{A. Data collection}

A force sensor, LUD-050-015-S*C01 (Loadstar sensors, US), was used to measure the grasping force. The sensors' capacity and accuracy are $50 \mathrm{lb}(22.68 \mathrm{~kg})$ and $0.25 \%$, respectively. A customised software is designed to obtain force data sampled at 25 samples/sec. A customised sEMG signal acquisition system was utilised to capture EMG signals from the forearm, as described in our previous work [17]. The EMG device owns 16 bi-polar channels with 3000 signal gain, 1 $\mathrm{kHz}$ sampling frequency and 12 bits ADC resolution. sEMG signals were restricted between $10 \mathrm{~Hz}$ and $500 \mathrm{~Hz}$ by a band 


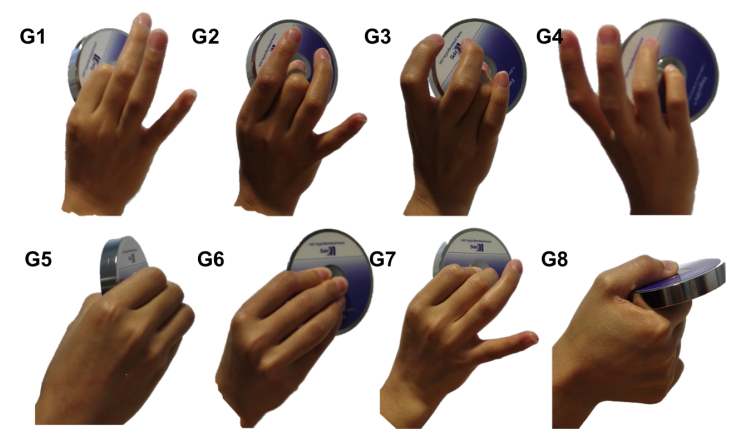

Fig. 4: Eight types of grasps

pass filter and the power line noises were filtered by a notch filter in hardware. All the electrodes were evenly distributed without muscle positioning but covered most forearm muscles.

To the best of our knowledge, it was the first attempt to use pinch force information to improve hand motion recognition accuracy, where evenly distributed EMG electrode configuration with $16 \mathrm{EMG}$ channels was utilized, as demonstrated in Fig. 3. It is not always a right choice of using large number of EMG channels, mainly because of limited space and high cost, although it is very likely to provide natural, reliable myoelectric control via increasing EMG channels [18]. Young, etc., [19] and $\mathrm{Li}$, etc. [20] presented that four to six channels were sufficient for pattern recognition based prosthetic manipulation. Thus, our experiments also consider the use of less numbers of EMG channel, 8-channel, 4-channel and 2-channel. When we talk about using 8-channel, channel 1 to 8 are selected. In the case of 4-channel, the $1 \mathrm{st}$, 3rd, 5 th and 7th channel are selected, while channel 1 and 5 are selected in the case of 2-channel.

Five subjects were employed to implement 8 types of grasps towards an object (i.e. the force sensor), as can be seen in Fig. 4. For each grasp, the subjects were asked to follow a changing given force that is linearly increases to $60 \%$ maximal voluntary contraction (MVC) in 10 seconds and decreases to $0 \% \mathrm{MVC}$ in the next 10 seconds. This procedure was repeated 3 times for each grasp.

\section{B. Feature Extraction}

Sliding window technique was utilised to extract the feature from EMG signal with a $200 \mathrm{~ms}$ window size and $40 \mathrm{~ms}$ increments. Consequently, the computing time for feature extraction and motion prediction should be less than $40 \mathrm{~ms}$. Four stable time domain features, root mean square (RMS), mean absolute value (MAV), zero crossings (ZC) and waveform length (WL) [5] and 4th-order aggressive regression (AR4) coefficients, were employed. These features has been widely accepted as the robust combination for EMG based motion recognition. Figs. 5(a) to 5(d) demonstrate an example of the force signal, raw EMG signal and the EMG features.

\section{Information Granulation and Classification}

This paper applied 10-fold-cross-test to evaluate the performance of FDGM via testing the data set from every subject

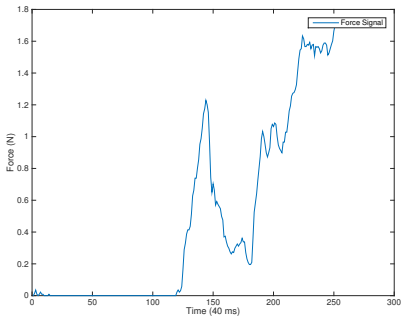

(a) Force Signal

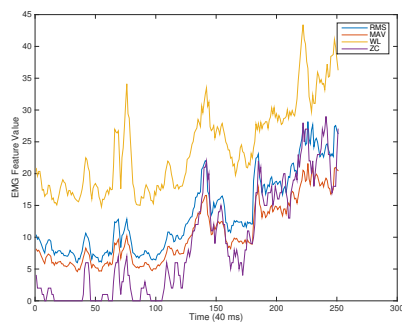

(c) 4 EMG features

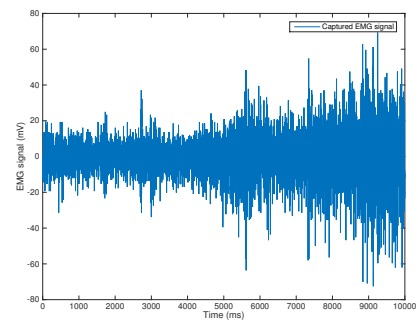

(b) Raw EMG signal

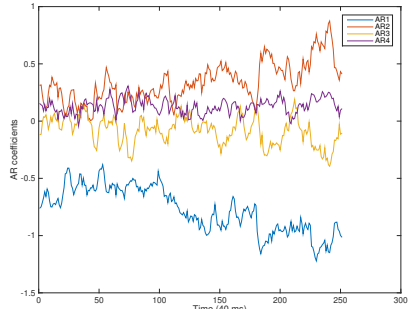

(d) AR coefficients
Fig. 5: Force signal, EMG signal and feature demonstration

by the following steps.

- Select 9 of 10 all observations as the training data set.

- Apply FDGM to generate $m$ granules for each class.

- Train the Fisher's LDA classifier to discriminate $8 * m$ classes.

- Predict class labels at the granular level from the remaining observations.

- Use the coarsening function to recover the predicted labels into 8 classes.

- Calculate the classification accuracy.

Above procedure was repeated 10 times to get the average accuracy.

\section{RESULT}

Three experiments were taken place to evaluate the performance of FDGM in EMG based grasp recognition. The first experiment compared traditional LDA with FDGM-based LDA in terms of classification accuracy. The second experiment evaluated the robustness of FDGM towards EMG signal interferences. The third experiment compared FDGM with other clustering based granular models in terms of accuracy and efficiency.

\section{A. LDA vs FDGM-based LDA}

The first experiment is to evaluate the effectiveness of FDGM in EMG based grasp recognition on the basis of conventional LDA classifier. The number of granules is optimised via Algorithm 1, where $N$ is set to 80 to satisfy the realtime performance in practical grasp recognition applications. Besides, it also ensures that each granule would contain sufficient samples for training. The convergence condition of Algorithm 1 is determined by $\left|\sum_{M-9}^{M-5}-\sum_{M-4}^{M}\right|<0.0001$, 
TABLE I: The comparison of FDGM-based LDA and conventional LDA in grasp recogntion (Error Rate \%)

\begin{tabular}{|c|c|c|c|c|c|c|c|c|}
\hline No. of Channels & \multicolumn{2}{|c|}{16} & \multicolumn{2}{c}{8} & 4 & 2 \\
\hline Methods & LDA & LDA_FD $\left(M_{o}\right)$ & LDA & LDA_FD $\left(M_{o}\right)$ & LDA & LDA_FD $\left(M_{o}\right)$ & LDA & LDA_FD( $\left.M_{o}\right)$ \\
\hline \hline Subject 1 & 0.65 & $0.07(33)$ & 2.46 & $0.61(47)$ & 12.74 & $8.47(77)$ & 27.9 & $23.2(55)$ \\
\hline Subject 2 & 2.75 & $1.03(18)$ & 6.86 & $3.44(31)$ & 12.33 & $7.31(77)$ & 26.98 & $23.63(74)$ \\
\hline Subject 3 & 0.74 & $0.14(59)$ & 2.03 & $0.55(63)$ & 9.74 & $5.17(76)$ & 23.81 & $19.63(46)$ \\
\hline Subject 4 & 0.57 & $0.13(18)$ & 1.94 & $0.95(67)$ & 6.26 & $4.55(30)$ & 20 & $18.46(67)$ \\
\hline Subject 5 & 2.69 & $1.46(24)$ & 6.95 & $3.37(62)$ & 13.14 & $8.04(69)$ & 37.77 & $34.53(13)$ \\
\hline \hline
\end{tabular}

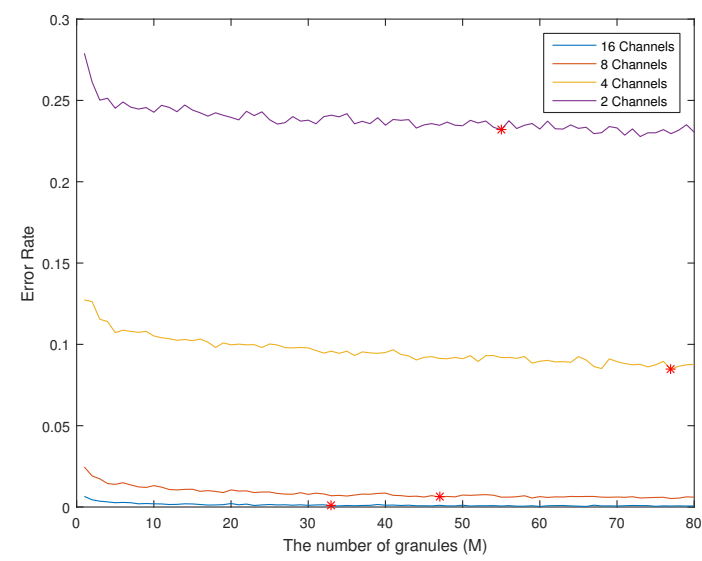

Fig. 6: The change of the error rate alone with the number of granules (Subject 1)

which indicates that the accuracy becomes stable. The experiment also considers different combinations of EMG channels, covering different practical situations where large number of electrodes can not be placed due to limited remaining space after amputation. Table I shows the comparison between LDA and FDGM-based LDA (LDA_FD) in terms of classification accuracy. Clearly, FDGM-based LDA outperforms LDA in achieving lower error rate. Taking the advantage of FDGM, the average error is reduced by $0.91 \%, 2.26 \%, 4.13 \%$ and $3.40 \%$ in 16-channel, 8-channel, 4-channel and 2-channel cases.

Moreover, the experiment also reveals that Algorithm 1 is able to optimise the number of granules, and provide an optimised one $\left(M_{o}\right)$. More commonly, it is also found that the error rate decreases along with the number of granules, as displayed in Fig. 6. The optimised number of granules are marked by red stars. The result also discloses that the error can always reduce dramatically by using several granules (less than 10 ), and then become relatively stable (very slow decrease). Based on the above findings, the left experiments apply FDGM by constructing 10 granules for each class.

\section{B. Sensitivity Analysis}

This experiment is to evaluate the stability of FDGM by means of adding synthetic white Gaussian noise (WGN) and $50 \mathrm{~Hz}$ power line noises to the captured EMG signals. The

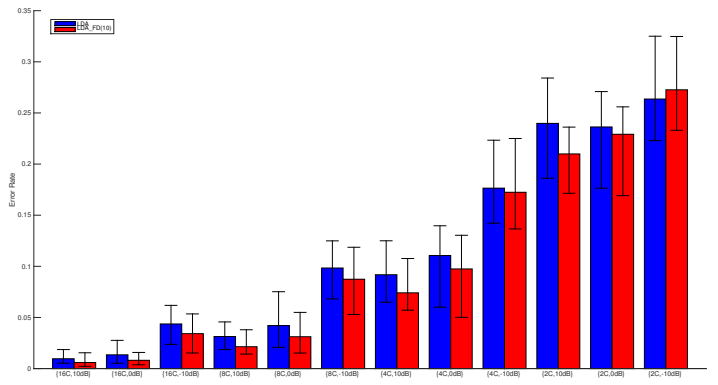

Fig. 7: The comparison of FDGM-based LDA and LDA with WGN interferences

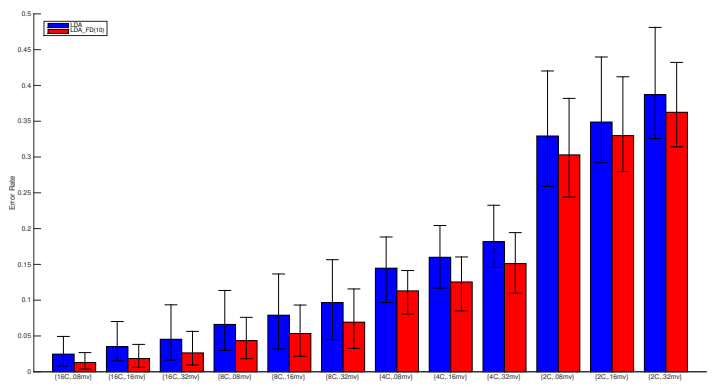

Fig. 8: The comparison of FDGM-based LDA vs LDA with power line noise interferences

intensity of Gaussian are measured by signal to noise ratio (SNR) with the unit of $\mathrm{dB} .10 \mathrm{~dB}, 0 \mathrm{~dB}$ and $-10 \mathrm{~dB}$ were tested. Fig. 7 demonstrates the average error across 5 subjects in four EMG channel combinations and three noise levels. It reveals that the FDGM is insensitive to WGN. All comparison groups show that the FDGM-based LDA performs better than conventional LDA, except when -10dB noise and 2-channel case are considered. It is possibly because the limited EMG information is overwhelmed by the noise. Similarly, $50 \mathrm{~Hz}$ power line noise was added to EMG signals with three levels of peak to peak voltages $(0.08 \mathrm{mv}, 0.16 \mathrm{mv}$ and $0.32 \mathrm{mv})$, and the result shows that FDGM is also robust towards power line noise, as seen in Fig. 8.

\section{FDGM vs other clustering based granular models}

This experiment compares FDGM with other clustering based granular models in terms of computing complexity 


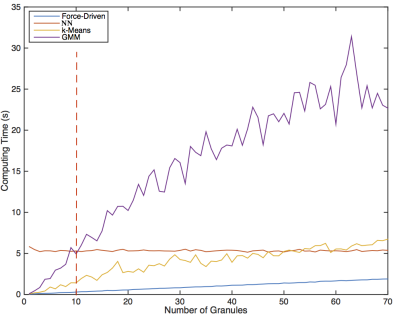

(a) Training time

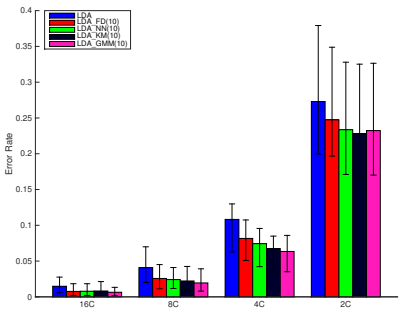

(b) Prediction error
Fig. 9: The comparison of granular models

and classification accuracy. The comparison group includes $\mathrm{NN}, \mathrm{k}-\mathrm{means}$ and GMM clustering algorithms. In terms of the training efficiency (Fig. 9(a)), FDGM requires the least training time among all the algorithms. Taking 10 granules as an example, the training time for FDGM is less than $0.5 \mathrm{~s}$, while NN, k-Means and GMM based granular model required about $5 \mathrm{~s}, 1.5 \mathrm{~s}$ and $5 \mathrm{~s}$ for training, respectively. In terms of the classification accuracy, four types of granular models achieved similar classification accuracy in 8- or 16-channel cases, while GMM outperformed the others in the 4-channel case and kmeans achieved the best in the 2-channel case. Taking both aspects into account, FDGM is very likely to achieve the best accuracy when the training time is constrained to less than 1 second. Moreover, it is worth to be noted again that FDGM owns better semantic interpretation than the others, and can be further exploited to estimate grasp force via checking the prediction result at the granular level.

\section{CONCLUSION AND FUTURE WORK}

FDGM is proposed in this paper to enhance the performance of EMG based grasp recognition. The proposed model utilised force information to implement the construction of granules during the training stage. Experimentally, this paper proved that 1) FDGM-based LDA is able to increase EMG based grasp recognition accuracy in comparison with conventional LDA; 2) FDGM-based LDA is insensitive to some practical EMG signal interferences; 3) FDGM is of the best training efficiency when compared with NN, k-means and GMM based granular models; 4) FDGM-based LDA shows a decreasing tendency along with the increase of granule number in a specific range; $5)$ the error decrease is more profound when the number of granules was less than 10 ; 6) 10 can be considered as the reference granule number in other similar studies, which could balance the accuracy and computation cost. Theoretically, FDGM emphasises the significance of semantics in the construction of granules, and thus the classification result at granular level could provide additional information. In our case, the predicted result contains the force information, and thus can be further extended to the application of grasping force estimation.

\section{ACKNOWLEDGMENT}

The contribution was funded by the 7th framework programme of the European Union (Grant No. 600915) and
National Natural Science Foundation of China (Grant Nos. 51575338, 51575407, 51475427).

\section{REFERENCES}

[1] E. Scheme and K. Englehart, "Electromyogram pattern recognition for control of powered upper-limb prostheses: State of the art and challenges for clinical use," J. Rehabil. Res. Dev., vol. 48, no. 6, pp. 643-659, 2011.

[2] A. H. Al-Timemy, G. Bugmann, J. Escudero, and N. Outram, "A preliminary investigation of the effect of force variation for myoelectric control of hand prosthesis," 35th Conf. Proc. IEEE Eng. Med. Biol. Soc., pp. 5758-5761, 2013.

[3] J. Y. He, D. G. Zhang, X. J. Sheng, S. C. Li, and X. Y. Zhu, "Invariant surface emg feature against varying contraction level for myoelectric control based on muscle coordination," IEEE J. Biomed. Health Inform., vol. 19, no. 3, pp. 874-882, 2015.

[4] M. Powell, R. R. Kaliki, N. V. Thakor et al., "User training for pattern recognition-based myoelectric prostheses: Improving phantom limb movement consistency and distinguishability," IEEE Trans. Neural Syst. Rehabil. Eng., vol. 22, no. 3, pp. 522-532, 2014.

[5] D. Tkach, H. Huang, and T. A. Kuiken, "Study of stability of timedomain features for electromyographic pattern recognition," J. Neuroeng. Rehabil., vol. 7, no. 1, p. 1, 2010.

[6] Y. Y. Yao, "Granular computing: basic issues and possible solutions," Proceedings of the Fifth Joint Conference on Information Sciences, Vols 1 and 2, pp. 186-189, 2000.

[7] H. Liu, C. Liu, and C. A. Wu, "Granular computing classification algorithms based on distance measures between granules from the view of set," Comput. Intell. Neurosci., vol. 2014, p. 656790, 2014.

[8] S.-B. Roh, W. Pedrycz, and T.-C. Ahn, "A design of granular fuzzy classifier,” Expert. Syst. Appl., vol. 41, no. 15, pp. 6786-6795, 2014.

[9] Y. Tang, B. Jin, Y. Sun, and Y.-Q. Zhang, "Granular support vector machines for medical binary classification problems," in Computational Intelligence in Bioinformatics and Computational Biology, 2004. CIBCB'04. Proceedings of the 2004 IEEE Symposium on. IEEE, pp. 73-78.

[10] M. L. Zhu and A. M. Martinez, "Subclass discriminant analysis," IEEE Trans. Pattern Anal. Mach. Intell., vol. 28, no. 8, pp. 1274-1286, 2006.

[11] L. A. Zadeh, "Toward a theory of fuzzy information granulation and its centrality in human reasoning and fuzzy logic," Fuzzy Set Syst., vol. 90, no. 2, pp. 111-127, 1997.

[12] S. Di Cataldo, A. Bottino, I. Ul Islam, T. F. Vieira, and E. Ficarra, "Subclass discriminant analysis of morphological and textural features for hep-2 staining pattern classification," Pattern Recogn., vol. 47, no. 7, pp. 2389-2399, 2014.

[13] S. W. Kim, "A pre-clustering technique for optimizing subclass discriminant analysis," Pattern Recogn. Lett., vol. 31, no. 6, pp. 462-468, 2010.

[14] A. Pnevmatikakis and L. Polymenakos, "Subclass linear discriminant analysis for video-based face recognition," J. Vis. Commun. Image R., vol. 20, no. 8, pp. 543-551, 2009.

[15] B. Mandal, L. Y. Li, V. Chandrasekhar, and J. H. Lim, "Whole space subclass discriminant analysis for face recognition," 2015 IEEE International Conference on Image Processing (Icip), pp. 329-333, 2015.

[16] J. T. Yao, A. V. Vasilakos, and W. Pedrycz, "Granular computing: perspectives and challenges," IEEE Trans. Cyb., vol. 43, no. 6, pp. 19771989, 2013.

[17] Y. Fang, H. Liu, G. Li, and X. Zhu, "A multichannel surface emg system for hand motion recognition," Int. J. Humanoid, p. 1550011, 2015.

[18] Y. Fang, N. Hettiarachchi, D. Zhou, and H. Liu, "Multi-modal sensing techniques for interfacing hand prostheses: A review," IEEE Sens. J, vol. 15, no. 11, pp. 6065-6076, 2015.

[19] A. J. Young, L. J. Hargrove, and T. A. Kuiken, "Improving myoelectric pattern recognition robustness to electrode shift by changing interelectrode distance and electrode configuration," IEEE Trans. Biomed. Eng., vol. 59, no. 3, pp. 645-652, 2012.

[20] N. Li, D. Yang, L. Jiang, H. Liu, and H. Cai, "Combined use of fsr sensor array and svm classifier for finger motion recognition based on pressure distribution map," J. Bionic Eng., vol. 9, no. 1, pp. 39-47, 2012. 